\title{
Tube-based distributed control of linear constrained systems
}

\author{
Stefano Riverso $^{\text {a }}$, Giancarlo Ferrari-Trecate ${ }^{\mathrm{a}}$ \\ ${ }^{a}$ Dipartimento di Ingegneria Industriale e dell'Informazione, Università degli Studi di Pavia, via Ferrata 1, 27100 Pavia, Italy
}

\begin{abstract}
In this paper we consider a linear system structured into physically coupled subsystems and propose an innovative distributed control scheme capable to guarantee asymptotic stability and satisfaction of constraints on system inputs and states. Our method hinges on the availability of a decentralized stabilizing regulator for the unconstrained system and provides a two-layer controller for each subsystem. Upper controllers receive planned state trajectories from neighboring subsystems and exploit the notion of tubes [12] for achieving robustness of stability with respect to coupling. Lower controllers generate planned trajectories using Model Predictive Control (MPC) independently of the other subsystems. The proposed control scheme is arguably easier to design and apply than existing distributed controllers with similar features. A comparison of the proposed approach with existing centralized and distributed MPC regulators is conducted using an illustrative example.
\end{abstract}

Key words: Distributed Control; Large-scale Systems; Robustness; Model Predictive Control; Decentralized Control.

\section{Introduction}

Modern engineering offers several examples of manmade systems characterized by a large number of states or deployed over a wide area. In these cases centralized control schemes, where all units are linked with a single multivariable regulator, can be unpractical or even prohibitive for several reasons including the cost of communication, the burden for computing simultaneously all control actions and the limited scalability of the controller if parts of the plant are added, removed or replaced.

An alternative approach is offered by decentralized control where the plant is decomposed into physically coupled subsystems regulated independently. The key issue, studied since the seventies, is how to design local controllers guaranteeing stability and suitable performance levels for the collective systems [13]. In decentralized control, regulators associated to subsystems do not exchange pieces of information and this can result in conservative control schemes when constraints on input and

\footnotetext{
* This paper was not presented at any IFAC meeting. The research leading to these results has received funding from the European Union Seventh Framework Programme [FP7/2007-2013] under grant agreement $\mathrm{n}^{\circ} 257462$ HYCON2 Network of excellence.

Email addresses: stefano.riverso@unipv.it (Stefano Riverso), giancarlo.ferrari@unipv.it (Giancarlo Ferrari-Trecate).
}

states have to be taken into account. As an example, most existing decentralized MPC schemes $[9,14,8,23,1]$ rely on the assumption of small coupling between subsystems.

A compromise between centralized and decentralized control is offered by distributed controllers where information is transmitted in real time among regulators. An interesting issue is to understand if and how the exchange of signals can overcome some limitations of decentralized controllers. For constrained systems, Distributed MPC (DMPC) schemes have recently attracted the attention of many researchers and can be classified into cooperative and non-cooperative methods [19]. Cooperative DMPC schemes with stability guarantees have been proposed in [20] for linear systems and aim at approximating centralized MPC controllers. However, they require all-to-all communication between regulators and each MPC controller requires knowledge on how subsystem operations impact on the whole plant. Furthermore, existing solutions account for input constraints only with the exception of [4] where a DMPC scheme avoiding all-to-all communication and accounting for box constraints on inputs and outputs has been presented. Many other contributions $[3,5,21,7,6]$ focused on non-cooperative schemes where each MPC controller minimizes a performance index local to each subsystem. A recent approach [3] considered linear discretetime systems without constraints and proposed DMPC schemes guaranteeing stability of the origin under a specific controllability assumption. Remarkably, these 
methods assume transmission of information among neighboring subsystems only, i.e. subsystems that are dynamically coupled. Neighbor-to-neighbor communication is also exploited in [5] where a DMPC regulator for non-linear continuous-time systems subject to input constraints has been proposed. A robust DMPC scheme for subsystems coupled only through constraints has been recently proposed in [21]. To date, the most featurerich non-cooperative DMPC schemes for discrete-time linear systems have been proposed in $[7,6]$. Relying on the existence of a decentralized state-feedback static stabilizing controller for the unconstrained system, the controllers in $[7,6]$ account for constraints on state and inputs of individual subsystem as well as coupling constraints among states of different subsystems. Moreover, communication is required only among neighboring subsystems or subsystems involved in state coupling constraints. Stability of the closed-loop system can then be guaranteed under suitable weak assumptions.

In this paper, we propose a new distributed control scheme for linear discrete-time systems dynamically coupled and input decoupled, with local state and input constraints and global state constraints. We first propose a standard procedure for turning a state-feedback stabilizing decentralized controller (that can be computed using the procedures reviewed in [13] and [23]) into a stabilizing distributed controller. Our method is based on the notion of tubes proposed in [12] for developing computationally affordable, robust MPC schemes and used in $[21,7,6]$ for designing DMPC regulators. Here we extend the approach of $[7,6]$ in order to propose local controllers that have a hierarchical structure. The upper controller $\mathcal{U C}_{i}$ (see Figure 1) for subsystem $i$ exploits transmitted information from neighboring controllers and it is coupled with a lower level controller, independent of neighboring subsystems, that allows input and state constraints of the whole system to be fulfilled.

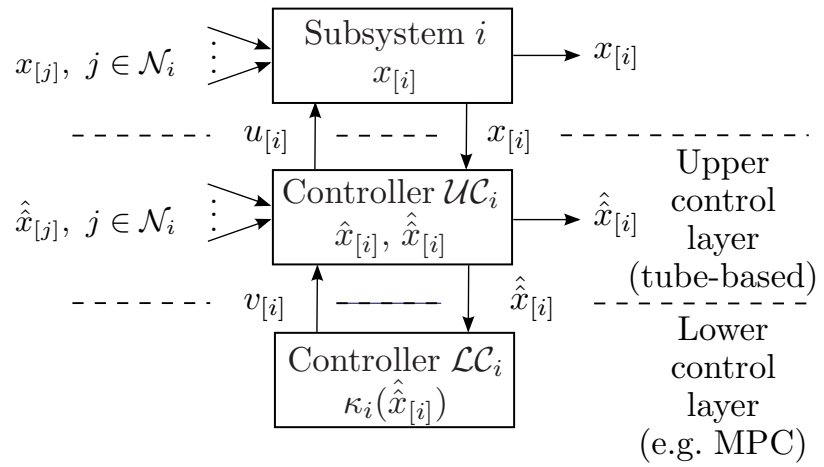

Fig. 1. Subsystem $i$ equipped with the upper controller $\mathcal{U C}_{i}$ and lower controller $\mathcal{L C}_{i}$.

While we adopt the same settings of $[7,6]$ the main advantages of our method are: (1) simpler initialization of the controller; (2) reduced amount of transmitted variables between subsystems in each sampling interval; (3) existence of a non-trivial region of attraction of the origin independently of the coupling strength among subsystems; (4) the possibility of using standard explicit MPC techniques [2] to compute local MPC regulators. There are also several differences between our DMPC scheme and the one proposed in [21]. The most important one is that our method applies to physically coupled subsystems.

The paper is structured as follows. The design of upper and lower controllers is introduced in Section 2 with a focus on the properties guaranteeing asymptotic stability of the origin and constraint satisfaction. In Section 3 we discuss how to compute all quantities local controllers depend upon. In Section 4 the distributed control scheme is applied to an example system and Section 5 is devoted to concluding remarks.

Notation. We use $a$ : $b$ for the set of integers $\{a, a+1, \ldots, b\}$. The column vector with $s$ components $v_{1}, \ldots, v_{s}$ is $\mathbf{v}=\left(v_{1}, \ldots, v_{s}\right)$. The function $\operatorname{diag}\left(G_{1}, \ldots, G_{s}\right)$ denotes the block-diagonal matrix composed by $s$ block $G_{i}, i=1, \ldots, s$. The symbol $\oplus$ denotes the Minkowski sum, i.e. $A=B \oplus C$ iff $A=\{a: a=b+c$, for all $b \in B$ and $c \in C\}$. Moreover, $\bigoplus_{i=1}^{s} G_{i}=G_{1} \oplus \ldots \oplus G_{s}$. The Pontryagin difference is denoted by $\ominus$, i.e. $A=B \ominus C$ iff $A=\{a: a+c \in B, \forall c \in C\}$. The symbol $\|\cdot\|$ is the Euclidean norm in $\mathbb{R}^{n}$. We also use $\|x\|_{P}$ to define the $P$-weighted seminorm, defined for all $x \in \mathbb{R}^{n}$ by $\|x\|_{P}{ }^{2}=x^{T} P x$, where $P$ is positive-semidefinite real symmetric matrix.

The set $\mathbb{X} \subseteq \mathbb{R}^{n}$ is Robust Positively Invariant (RPI) [17] for $x(t+1)=f(x(t), w(t)), w(t) \in \mathbb{W} \subseteq \mathbb{R}^{m}$ if $x(t) \in \mathbb{X} \Rightarrow f(x(t), w(t)) \in \mathbb{X}, \forall w(t) \in \mathbb{W}$. The RPI set $\mathbb{X}$ is minimal if every other RPI $\tilde{\mathbb{X}}$ verifies $\tilde{\mathbb{X}} \supseteq \mathbb{X}$.

\section{Decentralized-based distributed control of linear systems}

\subsection{System definition}

We consider a discrete-time Linear Time-Invariant (LTI) system

$$
\mathbf{x}^{+}=\mathbf{A} \mathbf{x}+\mathbf{B u}
$$

where $\mathbf{x} \in \mathbb{R}^{n}$ and $\mathbf{u} \in \mathbb{R}^{m}$ are the state and the input, respectively, at time $t$ and $\mathbf{x}^{+}$stands for $\mathbf{x}$ at time $t+1$. We will use the notation $\mathbf{x}(t), \mathbf{u}(t)$ only when necessary. The state is partitioned into $M$ state vectors $x_{[i]} \in \mathbb{R}^{n_{i}}$, $i \in \mathcal{M}=\{1, \ldots, M\}$ such that $\mathbf{x}=\left(x_{[1]}, \ldots, x_{[M]}\right)$, and $n=\sum_{i \in \mathcal{M}} n_{i}$. Similarly, the input is partitioned into $M$ vectors $u_{[i]} \in \mathbb{R}^{m_{i}}, i \in \mathcal{M}$ such that $\mathbf{u}=\left(u_{[1]}, \ldots, u_{[M]}\right)$ and $m=\sum_{i \in \mathcal{M}} m_{i}$.

We assume the dynamics of the $i$-th subsystem is given by

$$
x_{[i]}^{+}=A_{i i} x_{[i]}+\sum_{j \in \mathcal{N}_{i}} A_{i j} x_{[j]}+B_{i} u_{[i]}
$$


where $A_{i j} \in \mathbb{R}^{n_{i} \times n_{j}}, i, j \in \mathcal{M}, B_{i} \in \mathbb{R}^{n_{i} \times m_{i}}$ and $\mathcal{N}_{i}$ is the set of neighbors to subsystem $i$ defined as $\mathcal{N}_{i}=\{j \in$ $\left.\mathcal{M}: A_{i j} \neq 0, i \neq j\right\}$. According to (2), the matrix $\mathbf{A}$ in (1) is decomposed into blocks $A_{i j}, i, j \in \mathcal{M}$. We also define $\mathbf{A}_{\mathbf{D}}=\operatorname{diag}\left(A_{11}, \ldots, A_{M M}\right)$ and $\mathbf{A}_{\mathbf{C}}=\mathbf{A}-\mathbf{A}_{\mathbf{D}}$, i.e. $\mathbf{A}_{\mathbf{D}}$ collects the state transition matrices of every subsystem and $\mathbf{A}_{\mathbf{C}}$ collects coupling terms between subsystems. From (2) one also obtains $\mathbf{B}=\operatorname{diag}\left(B_{1}, \ldots, B_{M}\right)$ because submodels (2) are input decoupled.

\subsection{The upper control layer}

In this Section we discuss how it is possible to build a stabilizing distributed controller for system (1) based on the availability of a state-feedback, non-dynamical, stabilizing and decentralized controller, i.e. a matrix $\mathbf{K}=$ $\operatorname{diag}\left(K_{1}, \ldots, K_{M}\right), K_{i} \in \mathbb{R}^{m_{i} \times n_{i}}, i \in \mathcal{M}$ such that $\mathbf{A}+\mathbf{B K}$ is Schur. To this purpose we first clarify the exchange of information among subsystems. At time $t$ each subsystem receives a planned state $\hat{\hat{x}}_{[i]} \in \mathbb{R}^{n_{i}}$ from its neighbors. Then, the controller associated to system $i$ uses the measured states $x_{[i]}$ and $\hat{\hat{x}}_{[j]}, j \in \mathcal{N}_{i}$ for computing the control input $u_{[i]}$. The dynamics (2) of subsystem $i$ can be written as

$$
\begin{aligned}
x_{[i]}^{+} & =A_{i i} x_{[i]}+B_{i} u_{[i]}+\sum_{j \in \mathcal{N}_{i}} A_{i j} \hat{\hat{x}}_{[j]}+w_{[i]} \\
w_{[i]} & =\sum_{j \in \mathcal{N}_{i}} A_{i j}\left(x_{[j]}-\hat{\hat{x}}_{[j]}\right)
\end{aligned}
$$

As in $[7,6]$, in the spirit of tube-based control, we treat $w_{[i]}$ as a disturbance and define the nominal model

$$
\begin{aligned}
& \hat{x}_{[i]}^{+}=A_{i i} \hat{x}_{[i]}+B_{i} \hat{u}_{[i]}+\hat{w}_{[i]} \\
& \hat{w}_{[i]}=\sum_{j \in \mathcal{N}_{i}} A_{i j} \hat{\hat{x}}_{[j]}
\end{aligned}
$$

where $\hat{u}_{[i]} \in \mathbb{R}^{m_{i}}$ is the input and $\hat{x}_{[i]} \in \mathbb{R}^{n_{i}}$ is the state. Furthermore, we assume

$$
u_{[i]}=\hat{u}_{[i]}+K_{i}\left(x_{[i]}-\hat{x}_{[i]}\right) .
$$

Note that the planned states $\hat{\hat{x}}_{[j]}$ act as coupling terms in (5). Then, differently from $[7,6]$, we exploit once more tube-based control and treat $\hat{w}_{[i]}$ in (5) as a disturbance hence defining the system

$$
\hat{\hat{x}}_{[i]}^{+}=A_{i i} \hat{\hat{x}}_{[i]}+B_{i} v_{[i]}
$$

where $v_{[i]} \in \mathbb{R}^{m_{i}}$ is the input and $\hat{\hat{x}}_{[i]} \in \mathbb{R}^{n_{i}}$ is the state. Moreover, for $\hat{K}_{i} \in \mathbb{R}^{m_{i} \times n_{i}}$ we set in (5)

$$
\hat{u}_{[i]}=v_{[i]}+\hat{K}_{i}\left(\hat{x}_{[i]}-\hat{\hat{x}}_{[i]}\right)
$$

From (5)-(9), we obtain the upper controller $\mathcal{U C}_{i}$

$$
\mathcal{U C}_{i}:\left\{\begin{array}{c}
\hat{x}_{[i]}^{+}=\left(A_{i i}+B_{i} \hat{K}_{i}\right) \hat{x}_{[i]}+\sum_{j \in \mathcal{N}_{i}} A_{i j} \hat{\hat{x}}_{[j]}- \\
-B_{i} \hat{K}_{i} \hat{\hat{x}}_{[i]}+B_{i} v_{[i]} \\
\hat{\hat{x}}_{[i]}^{+}=A_{i i} \hat{\hat{x}}_{[i]}+B_{i} v_{[i]} \\
u_{[i]}=v_{[i]}+\hat{K}_{i}\left(\hat{x}_{[i]}-\hat{\hat{x}}_{[i]}\right)+K_{i}\left(x_{[i]}-\hat{x}_{[i]}\right)
\end{array}\right.
$$

which is driven by the exogenous input $v_{[i]}$. Note that the only pieces of information from neighboring subsystems used by $\mathcal{U C}_{i}$ are the planned states $\hat{\hat{x}}_{[j]}$ (see Figure 1 ). This reveals the distributed nature of controllers $\mathcal{U C}_{i}$. We also highlight that (8) defines the dynamics of the planned states.

Next, we clarify properties of matrices $K_{i}$ and $\hat{K}_{i}, i \in$ $\mathcal{M}$, that are required for the stability of system (3)-(9). Defining the errors

$$
\begin{aligned}
& z_{[i]}=x_{[i]}-\hat{x}_{[i]} \\
& \hat{z}_{[i]}=\hat{x}_{[i]}-\hat{x}_{[i]}
\end{aligned}
$$

from (3)-(9) one obtains

$$
\begin{aligned}
z^{+}{ }_{[i]} & =\left(A_{i i}+B_{i} K_{i}\right) z_{[i]}+\sum_{j \in \mathcal{N}_{i}} A_{i j} z_{[j]}+\sum_{j \in \mathcal{N}_{i}} A_{i j} \hat{z}_{[j]} \\
\hat{z}_{[i]}^{+} & =\left(A_{i i}+B_{i} \hat{K}_{i}\right) \hat{z}_{[i]}+\hat{w}_{[i]} .
\end{aligned}
$$

Using the collective errors $\mathbf{z}=\left(z_{[1]}, \ldots, z_{[M]}\right) \in \mathbb{R}^{n}$ and $\hat{\mathbf{z}}=\left(\hat{z}_{[1]}, \ldots, \hat{z}_{[M]}\right) \in \mathbb{R}^{n}$, from $(6),(8),(13)$ and $(14)$ one has

$$
\left[\begin{array}{c}
\mathbf{z} \\
\hat{\mathbf{z}} \\
\hat{\hat{\mathbf{x}}}
\end{array}\right]^{+}=\left[\begin{array}{ccc}
\mathbf{A}+\mathbf{B K} & \mathbf{A}_{\mathbf{C}} & \mathbf{0} \\
\mathbf{0} & \mathbf{A}_{\mathbf{D}}+\mathbf{B} \hat{\mathbf{K}} & \mathbf{A}_{\mathbf{C}} \\
\mathbf{0} & \mathbf{0} & \mathbf{A}_{\mathbf{D}}
\end{array}\right]\left[\begin{array}{c}
\mathbf{z} \\
\hat{\mathbf{z}} \\
\hat{\hat{\mathbf{x}}}
\end{array}\right]+\left[\begin{array}{c}
\mathbf{0} \\
\mathbf{0} \\
\mathbf{B}
\end{array}\right] \mathbf{v} .
$$

This system has a cascade structure in the sense that $\mathbf{v}$ acts only on $\hat{\hat{\mathbf{x}}}, \hat{\hat{\mathbf{x}}}$ acts only on $\hat{\mathbf{z}}$ and $\hat{\mathbf{z}}$ acts only on $\mathbf{z}$. Noting that $\mathbf{A}_{\mathbf{D}}+\mathbf{B} \hat{\mathbf{K}}=\operatorname{diag}\left(A_{11}+B_{1} \hat{K}_{1}, \ldots, A_{M M}+\right.$ $\left.B_{M} \hat{K}_{M}\right)$, the following assumption must be fulfilled for stability.

Assumption 1 The matrices $\mathbf{A}+\mathbf{B K}$ and $A_{i i}+B_{i} \hat{K}_{i}$, $i \in \mathcal{M}$ are Schur.

It is easy to show that if subsystems are decoupled, i.e. $A_{i j}=0, i \neq j$, under Assumption 1 the system given by (3) and (7) is a follower of system (5) and the system given by (5) and (9) is a follower of system (8), in the sense that $\mathbf{x}(t)-\hat{\mathbf{x}}(t) \rightarrow 0$ and $\hat{\mathbf{x}}(t)-\hat{\hat{\mathbf{x}}}(t) \rightarrow 0$ as $t \rightarrow+\infty$. Therefore, the planned states $\hat{\hat{x}}_{[i]}$ can be interpreted as references that states $x_{[i]}$ try to follow. 


\subsection{The lower control layer}

In this Section we show how controllers $\mathcal{U C}_{i}$ enable the design of a lower control layer capable to stabilize system (1) while fulfilling state and input constraints. More in detail, we equip subsystems $i \in \mathcal{M}$ with the constraints $x_{[i]} \in \mathbb{X}_{i}, u_{[i]} \in \mathbb{U}_{i}$ where $\mathbb{X}_{i}$ and $\mathbb{U}_{i}$ contain the origin in their interior, and define the sets $\mathcal{X}=\prod_{i \in \mathcal{M}} \mathbb{X}_{i}, \mathbb{U}=$ $\prod_{i \in \mathcal{M}} \mathbb{U}_{i}$. We also allow for collective state constraints given by $\mathbb{C}=\left\{\mathbf{x} \in \mathbb{R}^{n}: H(\mathbf{x}) \leq 0\right\}$ for a suitable function $H(\mathbf{x}): \mathbb{R}^{n} \rightarrow \mathbb{R}^{c}$ and assume the origin is in the interior of $\mathbb{C}$. Then, setting $\mathbb{X}=\mathcal{X} \cap \mathbb{C}$, we consider the collective constrained system (1) with

$$
\mathbf{x} \in \mathbb{X}, \mathbf{u} \in \mathbb{U}
$$

Our next goal is to design state-feedback non-dynamical lower controllers $v_{[i]}=\kappa_{i}\left(\hat{\hat{x}}_{[i]}\right)$ such that the origin of the closed-loop system (3)-(9) is asymptotically stable and constraints (16) are fulfilled at all time instants. For constraint satisfaction, as in tube-based control, we will compute tightened constraints $\hat{\mathbb{X}} \subseteq \mathbb{X}$ and $\mathbb{V} \subseteq \mathbb{U}$ such that,

$$
\begin{aligned}
& \hat{\hat{x}}_{[i]}(k) \in \hat{\hat{\mathbb{X}}}_{i}, v_{[i]}(k) \in \mathbb{V}_{i}, \forall i \in \mathcal{M}, \forall k \in 0: t \\
& \quad \Rightarrow \mathbf{x}(k) \in \mathbb{X}, \mathbf{u}(k) \in \mathbb{U}, \forall k \in 0: t
\end{aligned}
$$

Then we will require $\hat{\hat{x}}_{[i]}(0) \in \hat{\hat{\mathbb{X}}}_{i}$ and that lower controllers fulfill the following assumption.

\section{Assumption 2 Lower controllers}

$$
\mathcal{L C}_{i}: v_{[i]}=\kappa_{i}\left(\hat{\hat{x}}_{[i]}\right)
$$

guarantee $\hat{\hat{x}}_{[i]}^{+} \in \hat{\hat{\mathbb{X}}}_{i}, v_{[i]} \in \mathbb{V}_{i}, \forall i \in \mathcal{M}$.

We start characterizing constraints on $\mathbf{x}$ and $\mathbf{u}$ induced by arbitrary constraints $\hat{\hat{\mathbb{X}}}_{i}$ and $\mathbb{V}_{i}$ on $\hat{\hat{x}}_{[i]}$ and $v_{[i]}$, respectively.

Assumption 3 Sets $\hat{\mathbb{X}}_{i}$ and $\mathbb{V}_{i}, i \in \mathcal{M}$, are compact and contain the origin in their interior.

From the results in [10], under Assumptions 1 and 3 there exist nonempty RPIs $\hat{\mathbb{Z}}_{i} \subseteq \mathbb{R}^{n_{i}}, i \in \mathcal{M}$ for (14) and $\hat{w}_{i} \in \hat{\mathbb{W}}_{i}=\bigoplus_{j \in \mathcal{N}_{i}} A_{i j} \hat{\hat{\mathbb{X}}}_{j}$. Moreover, there is a nonempty $\mathrm{RPI} \mathbb{Z} \subseteq \mathbb{R}^{n}$ for

$$
\mathbf{z}^{+}=(\mathbf{A}+\mathbf{B K}) \mathbf{z}+\mathbf{w}
$$

and $\mathbf{w} \in \mathbb{W}=\mathbf{A}_{\mathbf{C}} \hat{\mathbb{Z}}, \hat{\mathbb{Z}}=\prod_{i \in \mathcal{M}} \hat{\mathbb{Z}}_{i}$. We also define the following sets

$$
\begin{aligned}
& \overline{\mathbb{X}}=\hat{\mathbb{X}} \oplus \mathbb{Z}, \hat{\mathbb{X}}=\prod_{i \in \mathcal{M}} \hat{\mathbb{X}}_{i}, \hat{\mathbb{X}}_{i}=\hat{\hat{\mathbb{X}}}_{i} \oplus \hat{\mathbb{Z}}_{i} \\
& \overline{\mathbb{U}}=\mathbb{V} \oplus \hat{\mathbf{K}} \hat{\mathbb{Z}} \oplus \mathbf{K} \mathbb{Z}, \mathbb{V}=\prod_{i \in \mathcal{M}} \mathbb{V}_{i}
\end{aligned}
$$

and the collective vectors $\hat{\mathbf{x}}=\left(\hat{x}_{[1]}, \ldots, \hat{x}_{[M]}\right)$ and $\mathbf{u}=$ $\left(u_{[1]}, \ldots, u_{[M]}\right)$. The next Theorem establishes key properties of lower controllers.

Theorem 4 Let Assumptions 1, 2 and 3 hold and assume that for all $i \in \mathcal{M}$ controllers $\mathcal{L C}_{i}$ make the origin of (8) asymptotically stable with region of attraction $\hat{\hat{\mathbb{X}}}_{i}^{a} \subseteq \hat{\hat{\mathbb{X}}}_{i}$. Then,

(a) the origin of the closed-loop system (3)-(9) is asymptotically stable;

(b) if the following conditions simultaneously hold

$$
\begin{aligned}
\hat{\hat{x}}_{[i]}(0) & \in \hat{\hat{\mathbb{X}}}_{i}^{a}, i \in \mathcal{M} \\
\mathbf{x}(0)-\hat{\mathbf{x}}(0) & \in \mathbb{Z} \\
\hat{x}_{[i]}(0)-\hat{\hat{x}}_{[i]}(0) & \in \hat{\mathbb{Z}}_{i}, i \in \mathcal{M}
\end{aligned}
$$

$$
\begin{aligned}
\text { then }(\mathbf{x}(t), \hat{\mathbf{x}}(t), \hat{\hat{\mathbf{x}}}(t)) & \rightarrow 0 \text { as } t \rightarrow \infty \text { and constraints } \\
\mathbf{x}(t) & \in \overline{\mathbb{X}}, \mathbf{u}(t) \in \overline{\mathbb{U}}
\end{aligned}
$$

are fulfilled $\forall t \geq 0$.

PROOF. The proof can be found in the Appendix of [18].

In view of Theorem 4, for guaranteeing (16) we still have to solve the problem of computing sets $\hat{\mathbb{X}}_{i}$ and $\mathbb{V}_{i}, i \in \mathcal{M}$ verifying

$$
\overline{\mathbb{X}} \subseteq \mathbb{X}, \overline{\mathbb{U}} \subseteq \mathbb{U}
$$

Note that choosing $\hat{\mathbb{X}}_{i}=\{0\}$ and $\mathbb{V}_{i}=\{0\}, i \in \mathcal{M}$ one has $\overline{\mathbb{X}}=\{0\}$ and $\overline{\mathbb{U}}=\{0\}$ and hence (25) holds. However, in this case $(22)$ and (23) imply $x(0)=\hat{x}(0)=\hat{\hat{x}}(0)=$ 0 . It is therefore of interest to study when (25) can be fulfilled using sets $\hat{\hat{\mathbb{X}}}_{i}$ and $\mathbb{V}_{i}, i \in \mathcal{M}$ that contain the origin in their interior. The following result shows that such sets $\hat{\hat{X}}_{i}$ and $\mathbb{V}_{i}$ can be always found, irrespectively of the coupling strength among subsystems. Intuitively, this is possible because planned states, that are governed by lower controllers, modify the disturbances $\hat{w}_{[i]}$ and $w_{[i]}$ defined in (4) and (6), respectively. 
Proposition 5 If Assumption 1 holds, for given sets $\mathbb{X}$ and $\mathbb{U}$ containing the origin in their interior, there are sets $\hat{\mathbb{X}}_{i}$ and $\mathbb{V}_{i}, i \in \mathcal{M}$ verifying Assumption 3 and such that (25) holds.

PROOF. The proof can be found in [18].

\section{Practical design of the distributed control scheme}

In this section we discuss the following issues in the design of controllers $\mathcal{U C}_{i}$ and $\mathcal{L C}_{i}$ : how to compute sets $\hat{\mathbb{X}}_{i}$ and $\mathbb{V}_{i}, i \in \mathcal{M}$ for guaranteeing (25), how to design lower controllers (17) verifying the assumptions of Theorem 4 and how to compute $\hat{\mathbf{x}}(0)$ and $\hat{\hat{\mathbf{x}}}(0)$ verifying $(22)$ and (23) for a given $\mathbf{x}(0)$.

For the computation of sets $\hat{\hat{X}}_{i}$ and $\mathbb{V}_{i}, i \in \mathcal{M}$, we propose the procedure described in Algorithm 1 that has to be executed only once and off-line. Finite termination of

$\overline{\text { Algorithm } 1}$
$\mathbb{X}, \mathbb{U}$ and $\alpha>1$.

Output: updated sets $\hat{\hat{\mathbb{X}}}_{i}, \mathbb{V}_{i}$ and sets $\hat{\mathbb{Z}}_{i}, \mathbb{Z}$.

(I) Each subsystem $i \in \mathcal{M}$ computes $\hat{\mathbb{W}}_{i}=$ $\bigoplus_{j \in \mathcal{N}_{i}} A_{i j} \hat{\widehat{\mathbb{X}}}_{j}$ and $\hat{\mathbb{Z}}_{i}$ that is the minimal RPI for (14).

(II) Compute the following sets in a centralized fashion

(a) $\mathbb{W}=\mathbf{A}_{\mathbf{C}} \hat{\mathbb{Z}}, \hat{\mathbb{Z}}=\prod_{i \in \mathcal{M}} \hat{\mathbb{Z}}_{i}$

(b) $\tilde{\mathbb{Z}}$, the maximal RPI set for (18) such that $\tilde{\mathbb{Z}} \subseteq \mathbb{X} \ominus \hat{\hat{\mathbb{X}}} \ominus \hat{\mathbb{Z}}$ and $\mathbb{V} \oplus \hat{\mathbf{K}} \hat{\mathbb{Z}} \oplus \mathbf{K} \tilde{\mathbb{Z}} \subseteq \mathbb{U}$.

(III) If $\tilde{\mathbb{Z}} \neq \emptyset$ then set $\mathbb{Z}=\tilde{\mathbb{Z}}$ and stop;

otherwise set

$$
\hat{\hat{\mathbb{X}}}_{i} \leftarrow \hat{\hat{\mathbb{X}}}_{i} / \alpha, \mathbb{V}_{i} \leftarrow \mathbb{V}_{i} / \alpha
$$

and go to (I).

Algorithm 1 can be proved using arguments similar to the ones adopted in the proof of Proposition 5, that is provided in [18]. Note that if the algorithm stops then, from the definition of $\overline{\mathbb{X}}$ and $\overline{\mathbb{U}}$ in (19) and (20) one has that (25) is verified because of the inclusions in step (IIb). Outer approximations of minimal RPI sets in the step (I) and (II) can be computed with a given precision using the methods developed in [16]. Algorithms for computing $\tilde{\mathbb{Z}}$ in step (II-b) have been proposed in [11] when all sets are polytopes.
Algorithm 1 suffers from two main limitations. First, the shape of sets $\hat{\hat{\mathbb{X}}}_{i}$ and $\mathbb{V}_{i}$ provided as inputs might impact on the size of the output sets that have the same shape. Second, the computational bottleneck is the step (II) that involves the collective dynamics (18). Future research will consider the problem of distributing the computation of approximations of the set $\mathbb{Z}$ among subsystems, following, e.g., the approach in [15].

As for the synthesis of lower controllers (17), all assumptions of Theorem 5 concerning lower controllers can be fulfilled if $\kappa_{i}\left(\hat{\hat{x}}_{[i]}(t)\right)$ is the result of an MPC regulator, hereafter termed MPC- $i$, for system (8). For a review of MPC schemes with the desired properties we defer the reader to section 2.5.3.1 of [17].

Remark 6 The main source of conservatism of our $D M P C$ method is that sets $\overline{\mathbb{X}}$ and $\overline{\mathbb{U}}$, computed from the results of Algorithm 1, could be much smaller than $\mathbb{X}$ and $\mathbb{U}$, respectively. This is partially due to the fact that (i) tube-based control is a robust control technique that we used twice for counteracting the effect of coupling terms $\mathbf{w}$ and $\hat{\mathbf{w}}$; (ii) $M P C-i$ regulators do not use information from neighbors and the fulfilment of collective state constraints is achieved by shrinking the sets $\hat{\mathbb{X}}_{i}$ and $\mathbb{V}_{i}$ (and hence $\overline{\mathbb{X}}$ and $\overline{\mathbb{U}}$ ) in Algorithm 1. However, general statements are hard to make because $\overline{\mathbb{X}}$ and $\overline{\mathbb{U}}$ also depend upon the shape of $\mathbb{X}$ and $\mathbb{U}$ and the coupling terms $A_{i j}, i \neq j$. This suggests that a precise assessment of the degree of conservatism should be conducted on a case by case basis.

Next we address the problem of choosing the initial states $\hat{\mathbf{x}}(0)$ and $\hat{\hat{\mathbf{x}}}(0)$, given $\mathbf{x}(0)$ so as to verify $(21),(22)$ and (23). Many MPC- $i$ schemes guarantee that $\hat{\hat{\mathbb{X}}}_{i}^{a}$ is the set of states in $\hat{\hat{\mathbb{X}}}_{i}$ for which the MPC- $i$ optimization problem is feasible [17] and hence (21) can be replaced by

$$
\text { MPC- } i \text { problems are feasible for } \hat{\hat{x}}_{[i]}(0), \forall i \in \mathcal{M} \text {. }
$$

Conditions (22), (23) and (26) give rise to a bilevel programming problem $[22]$ in the unknowns $\hat{\mathbf{x}}(0)$ and $\hat{\hat{\mathbf{x}}}(0)$ that is usually hard to solve. However, when $\hat{\mathbb{X}}_{i}$ and $\mathbb{V}_{i}$ are polytopes, under suitable assumptions MPC schemes for linear constrained systems produce polytopic regions of attraction $\hat{\hat{\mathbb{X}}}_{i}^{a}$ that can be computed off-line in closed form by means of explicit MPC algorithms [2,11]. Furthermore, methods in [16] and [11] allow Algorithm 1 to produce sets $\hat{\mathbb{Z}}_{i}, i \in \mathcal{M}$ and $\mathbb{Z}$ that are polytopes. Therefore, if explicit MPC- $i$ regulators are used, (22), (23) and (26) amount to a feasibility problem that can be solved through linear programming. Another method for computing $\hat{\mathbf{x}}(0)$ and $\hat{\hat{\mathbf{x}}}(0)$ hinges on the observation that a 
stabilizing MPC- $i$ controller usually relies on the use of a terminal constraint set $\hat{\hat{\mathbb{X}}}_{f, i} \subseteq \hat{\hat{\mathbb{X}}}_{i}$ that is known in closed form and verifies $\hat{\hat{\mathbb{X}}}_{f, i} \subseteq \hat{\hat{\mathbb{X}}}_{i}$. Hence one can replace $(21)$ with the more restrictive condition $\hat{\hat{x}}_{[i]}(0) \in \hat{\hat{\mathbb{X}}}_{f, i}$ and, similarly to the previous case, solve a linear programming problem when sets $\hat{\mathbb{X}}_{f, i}, i \in \mathcal{M}$ are polytopes.

Remark 7 In terms of features, the DMPC scheme proposed in [7,6], is the closest among existing ones to our control strategy. However, substantial differences arise. First, in order to initialize our controller only the computation of states $\hat{x}(0)$ and $\hat{\hat{x}}(0)$ is required while, in [7,6] the user must supply initial assumed states over a whole control horizon. Note also that there is no systematic method for choosing initial planned trajectories in [7], while in our scheme the choice of initial states for the controller can be done using the procedures described above. Second, Theorem 4 and finite termination of Algorithm 1 show that tightened constraints for lower $M P C-i$ controllers guaranteeing satisfaction of constraints (16) can be always computed. Differently, in [7,6] the existence of suitable tightened constraints can be guaranteed only if coupling among subsystems is sufficiently weak. However, the DMPC scheme in [7,6], that uses tube-based control only once, might provide a region of attraction of the origin that is larger than the one produced by our controller, and therefore the choice of the most suitable control algorithm has to be conducted on the basis of the specific application at hand.

\section{Numerical example}

In this section, we apply the proposed DMPC scheme to the system proposed in [7] and illustrated in Figure 2. The system is composed by four trucks, with

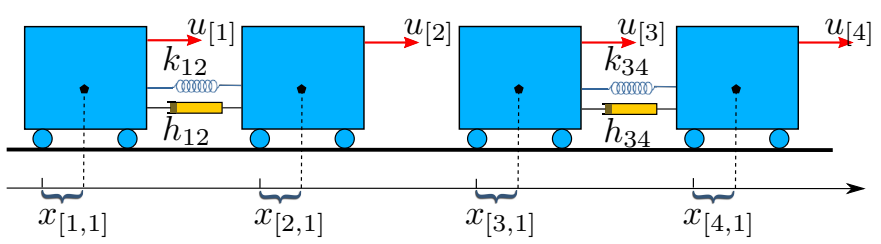

Fig. 2. Example system.

trucks 1-2 and 3-4 coupled by a spring and a damper. Parameters values, that are the same used in [7], are: $m_{1}=3, m_{2}=2, m_{3}=3, m_{4}=6, k_{12}=0.5, k_{34}=1$, $h_{12}=0.2, h_{34}=0.3$. Each truck $i \in \mathcal{M}=\{1,2,3,4\}$, is a subsystem with state variables $x_{[i]}=\left(x_{[i, 1]}, x_{[i, 2]}\right)$ and input $u_{[i]}$, where $x_{[i, 1]}$ is the displacement of truck $i$ with respect to a given equilibrium position, $x_{[i, 2]}$ is the velocity of the truck $i$ and $100 u_{[i]}$ is a force applied to truck $i$. Subsystems are equipped with the state constraints $\left|x_{[i, 1]}\right| \leq 4.5,\left|x_{[i, 2]}\right| \leq 2, i \in \mathcal{M}$ and with the input constraints $\left|u_{[i]}\right| \leq 1, i \in\{1,2,3\}$ and $\left|u_{[4]}\right| \leq 2$. Moreover, the collective constraints $\left|x_{[i, 1]}-x_{[i+1,1]}\right| \leq 6$, for $i=1,2,3$ are enforced.

The only neighbor of subsystem 1 is subsystem 2 (and vice-versa) because they are dynamically coupled. Similarly subsystem 3 and 4 are neighbors. The model has been discretized with sampling interval $\tau=0.1 \mathrm{~s}$.

In order to apply Algorithm 1, we define the decentralized controllers $\mathbf{K}=-\operatorname{diag}([0.535,0.253]$, [0.355,

0.168], [0.530, 0.252], [1.070, 0.507]) and $\hat{\mathbf{K}}=-\operatorname{diag}([0.103$, $0.112],[0.067,0.074],[0.098,0.111],[0.206,0.225])$ that guarantee fulfillment of Assumption 1.

For the following sets

$$
\begin{gathered}
\hat{\hat{\mathbb{X}}}_{i}=\left\{\left|\hat{\hat{x}}_{[i, 1]}\right| \leq 2.6,\left|\hat{\hat{x}}_{[i, 2]}\right| \leq 1.5\right\}, i \in \mathcal{M} \\
\mathbb{V}_{i}=\left\{\left|v_{[i]}\right| \leq 0.9\right\}, i=1,2,3 \quad \mathbb{V}_{4}=\left\{\left|v_{[4]}\right| \leq 1.9\right\}
\end{gathered}
$$

Algorithm 1 terminates in one iteration.

The lower controllers $\mathcal{L C}_{i}, i \in \mathcal{M}$, are synthesized using explicit MPC for system (8) based on the quadratic cost function

$$
\begin{gathered}
V_{i}^{N}\left(\hat{\hat{x}}_{[i]}(t), v_{[i]}\left(t: t+N_{i}-1\right)\right)= \\
\sum_{k=t}^{t+N_{i}-1}\left(\left\|\hat{\hat{x}}_{[i]}(k)\right\| Q_{Q_{i}}+\left\|v_{[i]}(k)\right\|_{R_{i}}\right)+\left\|\hat{\hat{x}}_{[i]}\left(t+N_{i}\right)\right\|_{S_{i}}
\end{gathered}
$$

where $N_{i}=10, i \in \mathcal{M}$,

$$
\begin{aligned}
Q_{1} & =\left[\begin{array}{cc}
0.120 & -0.014 \\
-0.014 & 0.144
\end{array}\right], Q_{2}=\left[\begin{array}{cc}
0.127 & -0.010 \\
-0.010 & 0.145
\end{array}\right], \\
Q_{3} & =\left[\begin{array}{cc}
0.120 & -0.014 \\
-0.014 & 0.144
\end{array}\right], Q_{4}=\left[\begin{array}{cc}
0.091 & -0.029 \\
-0.029 & 0.138
\end{array}\right], \\
R_{1} & =0.184, R_{2}=0.294, R_{3}=0.186, R_{4}=0.075
\end{aligned}
$$

and $S_{i}=\left[\begin{array}{ll}8.901 & 0.700 \\ 0.700 & 0.624\end{array}\right], i \in \mathcal{M}$. The matrices $Q_{i}, R_{i}$ and $S_{i}, i \in \mathcal{M}$ have been computed so as to guarantee stability of the origin of (8) and (17). Moreover they guarantee stability for the distributed MPC scheme proposed in [7]. Constraints for the MPC- $i$ problem are the dynamics $(8)$, state constraints $\hat{\hat{x}}_{[i]} \in \hat{\mathbb{X}}_{i}$, input constraints $v_{[i]} \in \mathbb{V}_{i}$ and suitable polytopic terminal constraints $\hat{\hat{\mathbb{X}}}_{f, i}$ for guaranteeing closed-loop stability of the origin of (8), see [17]. Explicit MPC- $i$ regulators have been computed using the MPT Toolbox [11] and then sets $\hat{\hat{\mathbb{X}}}_{i}^{a}$ are known. Initial states $\hat{\mathbf{x}}(0)$ and $\hat{\hat{\mathbf{x}}}(0)$ have been computed from $\mathbf{x}(0)$ solving a linear programming problem, as explained in Section 3. Figure 3 shows state trajectories obtained using a centralized MPC scheme, the distributed MPC method proposed in [7] and our distributed control scheme. In all cases, the initial state is $\mathbf{x}(0)=[1.8,0,-0.5,0,1,0,-1,0]^{T}$.

For centralized MPC, we have used the quadratic 

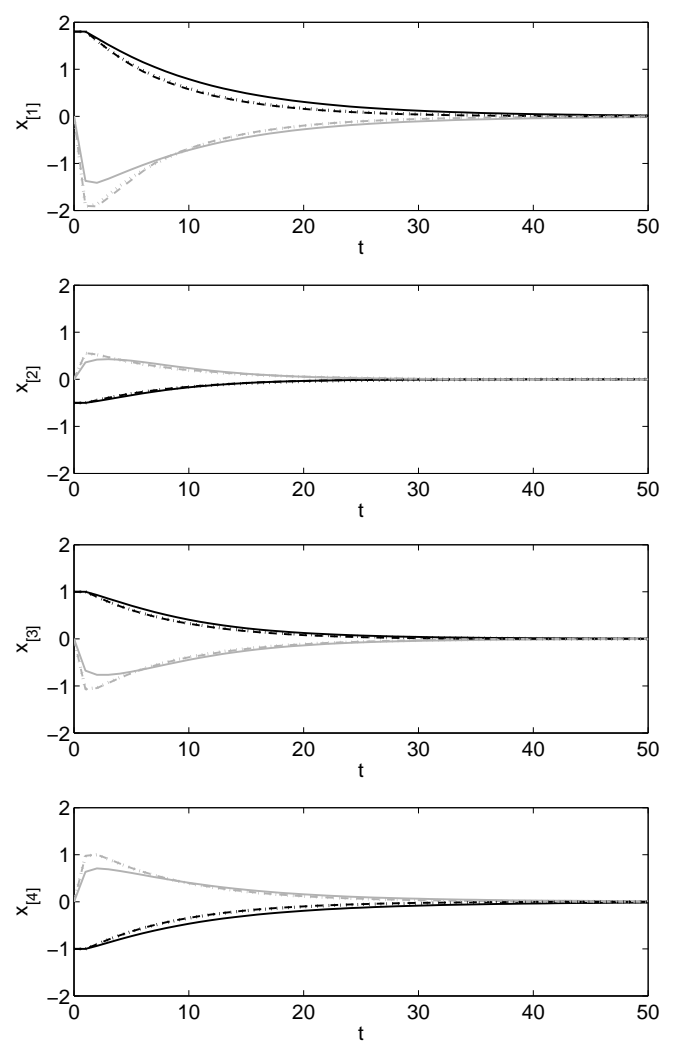

Fig. 3. State trajectories: positions (black) and velocities (grey) of the trucks, using centralized control (dotted lines), distributed control proposed in [7] (dashed lines) and our distributed control (solid lines).

cost function $V^{N}(\mathbf{x}(t), \mathbf{u}(t))=\sum_{k=t}^{t+N-1}\left(\|\mathbf{x}(k)\|_{\mathbf{Q}}+\right.$ $\left.\|\mathbf{u}(k)\|_{\mathbf{R}}\right)+\|\mathbf{x}(t+N)\|_{\mathbf{s}}$ where $N=10$ and $\mathbf{Q}=$ $\operatorname{diag}\left(Q_{1}, \ldots, Q_{M}\right), \mathbf{R}=\operatorname{diag}\left(R_{1}, \ldots, R_{M}\right)$ and $\mathbf{S}=$ $\operatorname{diag}\left(S_{1}, \ldots, S_{M}\right)$. These matrices guarantee stability of the origin of the collective system. Centralized MPC includes constraints on all states $x_{[i, j]}, i \in \mathcal{M}, j \in\{1,2\}$, on inputs $u_{[i]}$ and suitable terminal constraints for stability of the origin.

Figures 3 shows that the performance of our distributed control scheme is comparable with the performance of centralized MPC and the distributed control proposed in [7]. Table 1 highlights the computational advantages brought about by our method: since we can use standard explicit MPC for synthesizing local controllers, the average time for computing the inputs in each sampling interval is considerably reduced compared to the other methods ${ }^{1}$. Using the distributed MPC scheme proposed in [7], local optimization problems have a number of constraints comparable to the ones of centralized MPC. Moreover, since the local controllers of [7] depend of planned trajectories sent by the neighbors, explicit MPC

1 All simulations have been done using a processor Intel Core i5, $1.7 \mathrm{Ghz}$, Matlab r2011b, solver SDPT3 and MPT Toolbox [11]. methods cannot be applied out of the box. For these reasons, in our example computational savings brought about by the distributed MPC in [7] are limited.

\begin{tabular}{|c|c|c|c|c|c|}
\hline & \# Opt & Min Time & Mean Time & Max Time & Speed-up \\
\hline MPC & 50 & 0.3579 & 0.4577 & 0.5459 & 1 \\
\hline DMPC of [7] & 200 & 0.2581 & 0.3556 & 0.4866 & 1.29 \\
\hline Our DMPC & 0 & 0.0016 & 0.0017 & 0.0018 & 277.12 \\
\hline
\end{tabular}

\# Opt is the number of solved optimization problem. Min Time, Mean Time and Max Time represent respectively the minimum, the mean and the maximum time expressed in seconds to solve the optimization problem. Speed-up is obtained dividing the Mean Time of centralized MPC and the Mean Time each control scheme.

\section{Conclusions}

In this paper we proposed a novel DMPC scheme for linear constrained systems. We showed that the availability of a decentralized static state-feedback controller allows one to systematically design distributed controllers for each subsystem that stabilize the origin of the closedloop system and exchange only planned states at each sampling time. Furthermore, local controllers have a two-layer structure where MPC is exploited at the lower layer to guarantee constraint satisfaction. In the future we will study how to account for model uncertainties in the scheme and how to avoid off-line centralized computations in Algorithm 1. This will pave the way to methods for reconfiguring the controller on-line when subsystems are added or removed from the whole plant.

\section{Acknowledgements}

The authors are indebted with M. Farina and R. Scattolini for insightful discussions.

\section{References}

[1] A. Alessio, D. Barcelli, and A. Bemporad. Decentralized model predictive control of dynamically coupled linear systems. Journal of Process Control, 21(5):705-714, 2011.

[2] F. Borrelli. Constrained optimal control of linear and hybrid systems. Springer, Lecture Notes in Control and Information Sciences vol. 290, Berlin, Germany, 2003.

[3] E. Camponogara, D. Jia, B. H. Krogh, and S. Talukdar. Distributed model predictive control. IEEE Control Systems Magazine, 22(1):44-52, 2002.

[4] E. Camponogara and H. F. Scherer. Distributed Optimization for Model Predictive Control of Linear Dynamic Networks With Control-Input and Output Constraints. IEEE Transactions on Automation Science and Engineering, 8(1):233-242, 2011.

[5] W. B. Dunbar. Distributed Receding Horizon Control of Dynamically Coupled Nonlinear Systems. IEEE Transactions on Automatic Control, 52(7):1249-1263, 2007. 
[6] M. Farina and R. Scattolini. Distributed non-cooperative MPC with neighbor-to-neighbor communication. In Proceedings of the 18th IFAC World Congress, pages 404409, Milano, Italy, August 28 - September 2, 2011.

[7] M. Farina and R. Scattolini. Distributed predictive control: a non-cooperative algorithm with neighbor-toneighbor communication for linear systems. Technical report, Rapporto interno 2011.13, Dipartimento di Elettronica e Informazione, Politecnico di Milano, Milano, Italy, 2011.

[8] R. M. Hermans, M. Lazar, and A. Jokić. Almost decentralized Lyapunov-based nonlinear model predictive control. In Proceedings of American Control Conference 2010, pages 3932-3938, Baltimore, MD, USA, June 30 - July 02, 2010.

[9] T. Keviczky, F. Borrelli, and G. Balas. Decentralized receding horizon control for large scale dynamically decoupled systems. Automatica, 42(12):2105-2115, 2006.

[10] I. Kolmanovsky and E. G. Gilbert. Theory and computation of disturbance invariant sets for discrete-time linear systems. Mathematical Problems in Engineering, 4(4):317-363, 1998.

[11] M. Kvasnica, P. Grieder, and M. Baotić. Multi-Parametric Toolbox (MPT), 2004.

[12] W. Langson, I. Chryssochoos, S. V. Raković, and D. Q. Mayne. Robust model predictive control using tubes. Automatica, 40(1):125-133, 2004.

[13] J. Lunze. Feedback control of large scale systems. Prentice Hall, Systems and Control Engineering, Upper Saddle River, NJ, USA, 1992.

[14] D. M. Raimondo, L. Magni, and R. Scattolini. Decentralized MPC of nonlinear systems: An input-to-state stability approach. International Journal of Robust and Nonlinear Control, 17:1651-1667, 2007.

[15] S. V. Raković, B. Kern, and R. Findeisen. Practical Set Invariance for Decentralized Discrete Time Systems. In Proceedings of the 49th IEEE Conference on Decision and Control, pages 3283-3288, Hilton Atlanta Hotel, Atlanta, GA, USA, December 15-17, 2010.

[16] S. V. Raković, E. C. Kerrigan, K. I. Kouramas, and D. Q. Mayne. Invariant approximations of the minimal robust positively invariant set. IEEE Transactions on Automatic Control, 50(3):406-410, 2005.

[17] J. B. Rawlings and D. Q. Mayne. Model predictive control: theory and design. Nob Hill Pub., Madison, WI, USA, 2009.

[18] S. Riverso and G. Ferrari-Trecate. Tube-based distributed control of linear constrained systems. Technical report, RIDIS 156/12, Dipartimento di Informatica e Sistemistica, Università degli Studi di Pavia, Pavia, Italy, 2012.

[19] R. Scattolini. Architectures for distributed and hierarchical Model Predictive Control A review. Journal of Process Control, 19(5):723-731, 2009.

[20] B. T. Stewart, A. N. Venkat, J. B. Rawlings, S. J. Wright, and G. Pannocchia. Cooperative distributed model predictive control. Systems \& Control Letters, 59(8):460-469, 2010.

[21] P. Trodden and A. Richards. Distributed model predictive control of linear systems with persistent disturbances. International Journal of Control, 83(8):1653-1663, 2010.

[22] L. N. Vicente and P. H. Calamai. Bilevel and multilevel programming: A bibliography review. Journal of Global Optimization, 5(3):291-306, 1994.

[23] A. I. Zečević and D. D. Siljak. Control of complex systems: Structural constraints and uncertainty. Springer, Communications and Control Engineering, Berlin, Germany, 2010. 